\title{
Professional inclusion of people with schizophrenia: aspects of functionality and intervention strategies
}

\begin{abstract}
Description of professional experience of Social Worker in front of the Coordination of a professional inclusion program of people with schizophrenia in the job market through the Quotas Law. Presentation of the methodology of biopsychosocial evaluation on human functionality and impacts to the person with schizophrenia and the use of assistive technologies to support the promotion of inclusion in the labour market. The organization of data collected from 2016 to 2018, show the need to ensure clinical stability of people with schizophrenia and then insert them into the labour market. Identifying the type of constraints that exist allows for the targeting of jobs that meet the individuality of each individual and enhance their social skills. The proposed model for inclusion of people with schizophrenia in the job market is through vacancies aimed at people with mental or psychosocial disabilities - as has been approached in Brazil, the deficiencies related to mental disorders. Follow-up of steps such as: Screening (identification), Multidisciplinary assessments (based on CIF and ICD-10); Therapeutic Groups, Management aligned to the Unique Therapeutic Plan, Support and guidelines to the contracting company; as a Supported Employment strategy.
\end{abstract}

Keywords: work, inclusion, schizophrenia, mental disorder, quota law, labour
Volume 5 Issue 3 - 2018

\author{
Luciene Redondo de Freitas \\ PROESQ-Unifesp, Social Assistant (Unifesp), Brazil
}

Correspondence: Luciene Redondo de Freitas, PROESQUnifesp, Social Assistant (Unifesp), Consulting and Psychosocial Advisory, São Paulo, Brazil, Tel 55 + (I I) 97254-4255, Email luredon@yahoo.com.br

Received: June 05, 2018 | Published: June 20, 2018
Abbreviations: ICD, international classification of diseases; CIF, international classification of functioning; CLT, consolidation of labour laws; ILSS, independent living skills survey; ASD, autism spectrum disorder

\section{Introduction}

Schizophrenia affects about $0.4 \%$ of the world's population, in Brazil there would be about 800,000 people affected. Recent advances in treatment have allowed great success in controlling psychotic symptoms, but most persist with cognitive and functional difficulties. There is enormous difficulty in returning to social life, with impacts to the person with schizophrenia, their families and society. The Schizophrenia Program of the Paulista School of Medicine (PROESQ/ UNIFESP), created about 30 years ago, is a national and international reference in the development of technologies to assist the person with schizophrenia.

The INSERIR Program started in PROESQ in 2010, as a proposal to support patients who are stable in returning to the job market. Since then, intervention models have been developed and tested. The initial results indicated that it is very difficult for people with schizophrenia to stay on the job and, especially, to reach it in the formal market, that is, without being part of the Quota Law. People often omitted their state of mental health for fear of being prejudiced or not being accepted. Indeed, it is a fact, if we consider the stigma imposed on mental health and especially on people with schizophrenia.

In contact with the Supported Employment technology and the International Convention on the Rights of Persons with Disabilities, ${ }^{1}$ the INSERIR Program has returned its efforts since 2014 to effectively promote the professional inclusion of people with schizophrenia, combating stigma and fighting for his right to also have access to the
Quota Law, as a person with a psychosocial/mental disability, seeking partnerships with companies and public and private organizations, accompanying all stages of professional inclusion. As a social policy for access to work, the inclusion of people with severe mental disorders such as schizophrenia in the formal market offers the opportunity to generate sustainability, full autonomy and overcoming the social and attitudinal barriers imposed by the disorder.

Since then, reports (with ICD-10, CIF, neuropsychological) have been prepared with the support of all multi professional team with important data to the framework of the Law of Quotations and the knowledge of the employer and employment reference team supported on the singularity of each person with schizophrenia.

All persons included in the Program are followed for at least 12 months, through therapeutic groups, individual psychotherapy, and social-family counselling, psychiatric consultations, before and after the hiring process. Contracting companies are offered free ongoing support to managers and staff in case management and information on ongoing therapeutic follow-up, institutional visits, lectures, and relapse risk control. The present Professional Inclusion Program is the result of all construction and elaboration of the work plan of the PROESQ-Unifesp Social Worker, who as Coordinator started to manage all the necessary steps for the development of the Program as we will see next.

\section{Screening}

The enrolment of people in the Insert Program takes place through the evaluation in Triage, through referral of the multi professional team of PROESQ. In the Triage, it is sought to verify if the patient has the necessary requirements for joining the Program, which are:

a. Be over 18 years of age. 
b. Not be a beneficiary of retirement or sickness aid.

c. Have clinical stability for more than 6 months.

The minimum age established for admission was considered based on the patients followed up by PROESQ being exclusively above 18 years. Not being initially thought to include patients from other programs, such as PAPIA or Prisma. But in fact, there would be no impediment to inclusion of people under 18 years and with at least 14 years, for Young Apprentice condition (Law 10,097/00)

The restriction of access to the Insert Program for beneficiaries of Social Security, who receive pensions or are in sickness benefit scheme, is because all indications for job vacancies are in formal regime by the Consolidation of Labour Laws (CLT - Decree 5.452/43), which automatically when contracted, would exclude it from this benefit, preventing them from returning to the benefit and opposing the initial petition thereof. Since the new configuration of the work plan of the Insert Program, started in 2016, 94 people have already been evaluated in Screening. Of these, 24 people were disapproved in Triage, and only 4 people were due to be beneficiaries of retirement or sickness aid.

The evaluation of clinical stability in screening, considers data from the medical record and social interview with direct questions that identify:

a. Adherence to Treatment: response to therapeutic proposals, medication, clinical impression on the evolutionary summary of treatment and multi professional reports when they exist (Occupational Therapist, Neuropsychology, Psychological).

b. History of hospitalization: less than 6 months.

c. Critical of the disease: verified the existence of criticism (even if partial), on the part of the patient in relation to the treatment and diagnosis.

d. Assessment of autonomy and family support: patient's ability to walk alone in public spaces, ability to use public transportation, routine daily activities, family supports (protection or risk), social vulnerabilities.

Among the cases disapproved for lack of clinical stability in the period, there were identified cases where there were one or more situations and symptoms: Delusional speech, sexual hallucinations, bizarre behaviour, lack of critique of the disease, refusal of medication, severe obsessive disorder symptoms, lack of civil documentation (RG, CPF and workbook), social phobia, lack of autonomy to go and come, hostile behaviour, self-care greatly impaired, serious clinical problems.

The importance of establishing criteria for the enrolment of participants in the Insert Program is to guarantee lower risks of patient discontinuity in the Program or to achieve better results. Considering that the inclusion proposal is in the formal labour market, although through the Quota Law (Law 8,213/ 91 - article 93), rules and conduct of the company are established, in equal participation with other employees. Therefore, the care of the Insert Program with the requirement of "clinical stability" is in ensuring that the inclusion will be made with people who can be productive and functional in the workplace. We believe that there may be people with schizophrenia who are not ready at that time for a professional activity. Therefore, the evaluation also seeks to reorient the patient who is not absorbed in the Program, for other psychosocial interventions that may soon, facilitate the resumption of professional activities. For this reason, many patients are referred after screening for Occupational Therapy, Group Psychotherapy, Family Home Care, social-care services and referral in mental health near the home, and / or even Therapeutic Accompaniment.

\section{Admission}

After the approval of the patient in the Screening of the Insert Program, a therapeutic contract is established where it is informed about the operation of the Program clarifying its duties and rights, as follows:

\section{Program operation steps insert}

For a period of 12 months and free of charge:

a. Evaluations of functionality, professional profile, psychosocial assessment and medical report.

b. Therapeutic Groups.

c. Selective Processes.

d. Multi professional accompaniment (company, family and patient).

\section{Duties of the patient}

a. Appear in scheduled appointments: in the case of 3 consecutive absences, you may be disconnected from the Program.

b. Maintain treatment: if you refuse to continue treatment in PROESQ, you will be disconnected from the Program.

c. Communicate to the Insert Program, data of the employer company.

d. To authorize diagnostic information for requesting and enrolling in a vacancy for disabled people (Quota Law).

e. Keep updated contact information and home address.

\section{Patient rights}

a. Be guided on the return of any process of evaluation of functionality and professional profile.

b. Receive updated medical report.

c. To have support in the elaboration of curriculum and to send it to potential companies that can contract it.

d. Be accompanied by a professional of the Insert Program team in selective processes, interviews, and training or integration activities offered by the company.

e. Receive multi professional follow-up (Psychiatrist, Psychologist, Social Worker) regularly during their participation in the Program, as well as, intensively whenever necessary.

f. Receive support from the Insert Program team in dialogue with the human resources managers of the company in which they are working, favouring the accessibility and use of the same in place.

It is further clarified that the Insert Program does not guarantee the effective hiring of any of its participants. That is, actions are taken to promote professional inclusion, partnership with companies, but there is no guarantee that they will hire them, since they will be evaluated in selective processes with other people in the same situation or not, depending on the company and not from us, the approval and admission to work. Preferably, it is sought to establish the therapeutic 
contract in the presence of a relative of the patient, to strengthen the links with the Program and facilitate the clarification of any doubts.

Throughout the follow-ups, it is observed the importance of having the family as a partner in the process of professional inclusion of the patients. This is because a lot of them come to see the Insert Program team as one that will facilitate or bar their possibility of work, and it is not uncommon in some situations patients minimize to the team how they are feeling or leaving the company, and get information from very different family members, showing the need for more attention.

\section{Biopsychosocial evaluations}

The trajectory of evaluations proposed by the Insert Program, seeks to map the functional limitations that the participant with schizophrenia has, directing an individualized plan of therapeutic follow-up that favours professional inclusion and guarantees the framework of this, in the Quota Law, recognizing him as a person with mental or psychosocial disability. Since 1993, mental disorders have been inserted in the 10th. Revision of the International Classification of Diseases (ICD-10), since it is understood that diseases related to mental health are also the cause of aggravation of the functions of the individual's body and that in certain cases, the consequences of death, incapacity and performance limitation.

According to the World Health Organization, published in 2010, it is estimated that by 2020 , depression will be the main disease that will incapacitate people to work (The World Health Report 2001. Mental Health: New Understanding, New Hope). Currently, mental disorders are the main causes of suicide, incapacity for work and are directly related to many situations of violation of social rights and vulnerabilities. The $\mathrm{CIF}^{2}$ defines deficiencies as problems in functions (mental, sensory, voice, cardiovascular, haematological, respiratory, urinary, digestive, reproductive, etc.) or body structures, such as a major deviation or loss.

Thus, the term psychosocial deficiency is characterized by the classification of a problem in the mental functions of an individual, which may be one or more limitations of the capacities of consciousness, learning, social interaction, temperament, energy and impulse, attention, memory, concentration, language, perception, according to the classification of mental functions by the CIF. ${ }^{2}$ Its evaluation is made from the identification of the fragilities and limitations that the environmental ones provide, that is, the social and attitudinal barriers; and aspects of the structure and functions of the body and mind that, due to the existence of a mental disorder, directly affect the capacity of performance and functionality of the individual within the parameters of normality to the exercise of community life.

For this purpose, the Independent Living Skills Survey (ILSS) is used for the skills and autonomy inventory. It is important to emphasize that this scale was validated in Brazil by the Occupational Therapist Dr. Larissa Campagna, responsible for the idealization of the Insert Program in its first phase when it started in 2010. At that time, there was no specific action plan and intervention to reach but it was already sensitive to the need to provide space and listening for those interested in returning to the labour market, but who found difficulties. The use of the scale resulted in the reading of its results in accordance with the aspects of the functionality. Thus, after evaluating functionality, the functional report is made, with a $\mathrm{CIF}^{2}$ framework, by a qualified professional in the Insert Program for the elaboration of this program, which in this case has been done by the Coordinator and Social Worker.

In addition to this evaluation, there are also evaluations of occupational, socio-economic profile, existing social risks and vulnerabilities and identification of professional and psychological profile. It is known that psychosocial evaluation is provided by the Brazilian Inclusion Law, ${ }^{3}$ and according to Normative Instruction No. 98/2012 of the Ministry of Labour, it can be elaborated by any professional with Higher Education, specialized in the area of disability in question. The CIF-based report complements the medical report (ICD-10), with information that responds to the prerogatives of the law on the characterization of disability, namely:

(Law 13.146/15 - Art. 2) ${ }^{3}$ : A person with a disability is a person who has a long-term physical, mental, intellectual or sensorial disability, which, in interaction with one or more barriers, may obstruct their full participation and effective in society on equal terms with other people. $\S 1$ o the assessment of the disability, when necessary, will be biopsychosocial, carried out by a multidisciplinary and interdisciplinary team and will consider:

a. Impairments in the functions and structures of the body.

b. Socio-environmental, psychological and personal factors.

c. The limitation in the performance of activities.

d. The participation restriction.

The CIF report will therefore identify what are the barriers that the person with schizophrenia faces that make them be considered as persons with disabilities. That is, the identification of the existence or not of impediments in the functions and structures of the body, socio-environmental, psychological and personal factors that hinder access to rights in equal participation, limitations to the performance of activities.

The Program has adopted the practice of reporting in the CIF report, from 3 to 5 main aspects of barriers to the functionality of the participants. This is because it considers the need to clarify to the Occupational Medicine that will receive the award, the determining aspects of its framework for the Quotas Law, since there is still no established regulation of which mental disorder is or is not a psychosocial disability.

Unlike other disorders such as Autism Spectrum Disorder (ASD) and Down Syndrome, Schizophrenia does not have a unique legislation that recognizes it permanently as a disability. The Law of Quotas itself (Law No. 8,213/91) is urgent to keep up with the changes proposed by the UN Convention on the Rights of Persons with Disabilities, since it establishes, for example, that mental 18 years old, the person has overall intelligence and overall below average skill set.

For this reason, the Insertion Program has always sought in this new phase to reinforce the militancy of the subject "professional inclusion of people with schizophrenia, in the areas of defence and guaranteeing the rights of persons with disabilities." An important strategy that has shown its relevance and differential, since in many cases, we find little consensus with professionals of companies, that represent the Medicine of the Work or even, with Fiscal Auditors of the Ministry of the Work, on approving or not the schizophrenia framework in the Quota Law.

Proof of this is that since 2015 we are the only university participating in the São Paulo Chamber of Inclusion of Persons with Disabilities, developed by the Ministry of Labour and Employment of 
São Paulo and the only mental health equipment linked to the public health the exclusive representative of people with schizophrenia. A survey of 16 psychosocial assessments already performed presented a very specific picture of limitations of mental functions and of performance capacity for activities and social participation, as in Table $1 \&$ Table 2 .

Table 1 Functional Profile - Main body functions affected

Functional profile of program Participants insert, according to the international classification of functioning (CIF)

Sample: 16 cases Valuation Period: 2016 and 2017

$\mathrm{b}=$ body functions

(Identification of losses, deficits and limitations)

$\begin{array}{lll}\text { CIF } & \text { Amount } & \text { Reference } \\ \text { bI30 } & 6 & \text { power and boost functions } \\ \text { bI } 40 & \text { II } & \text { attention functions } \\ \text { bII4 } & 4 & \text { time and space orientation functions } \\ \text { bII0 } & 4 & \text { consciousness functions } \\ \text { bI } 44 & \text { I2 } & \text { memory functions } \\ \text { bI } 26 & 5 & \text { functions of temperament and personality } \\ \text { bI52 } & \text { I } & \text { emotional functions } \\ \text { bII7 } & 8 & \text { intellectual functions } \\ \text { bI } 22 & 20 & \text { global psychosocial functions (interpersonal skills) } \\ \text { bI56 } & 2 & \text { perception functions } \\ \text { bI60 } & 4 & \text { thought functions } \\ \text { bl64 } & 4 & \text { higher level cognitive functions } \\ \text { bI67 } & \text { I } & \text { mental language functions }\end{array}$

Table 2 Functionality profile - Main limitations of activities and participation

\begin{tabular}{|c|c|c|}
\hline \multicolumn{3}{|c|}{ Participant functionality profile of insert program } \\
\hline \multicolumn{3}{|c|}{$\begin{array}{l}\text { Sample: } 16 \text { cases Valuation Period: } 2016 \text { and } 2017 \\
d=\text { activities and participation } \\
\text { (Identification of losses, deficits and limitations) }\end{array}$} \\
\hline CIF & Amount & Reference \\
\hline dI70 & 1 & Write \\
\hline d720 & 1 & complex interpersonal interaction \\
\hline d330 & I & Speak \\
\hline d350 & I & Conversation \\
\hline dI77 & 2 & make decisions \\
\hline dI59 & I & basic learning \\
\hline dI99 & 2 & learning and application of knowledge \\
\hline $\mathrm{dI} 75$ & 8 & solve problems \\
\hline $\mathrm{d} 310$ & I & communicate and receive oral messages \\
\hline dI50 & I & learn to calculate \\
\hline $\mathrm{dl} 40$ & 2 & learn to read \\
\hline
\end{tabular}

\begin{tabular}{lll}
\hline CIF & Amount & Reference \\
d220 & 2 & perform multiple tasks \\
d520 & I & take care of body parts \\
dI60 & I & focus attention \\
dI10 & 3 & Observe \\
d640 & I & perform household chores \\
d315 & I & $\begin{array}{l}\text { communicate and receive non-verbal } \\
\text { d710 }\end{array}$ \\
I & basic interpersonal interactions \\
d750 & I & informal social relationships \\
d820 & I & schooling \\
d910 & I & community life \\
d450 & I & Floor \\
dI15 & I & Hear \\
\hline
\end{tabular}

According to these surveys presented in Tables $1 \&$ Table 2, the highest incidence of functional limitations of the evaluated participants is classified as impairment of global psychosocial mental functions (CIF b122), which are the mental functions necessary to constructively understand and integrate general mental functions, which lead to the formation of interpersonal skills necessary for the establishment of reciprocal social interactions in terms of meaning and purpose. Second, the second higher prevalence of functional limitations is related to short or long-term memory capacity (CIF b144), immediate, recent and remote memory, memory duration, functions used in recall and learning, as in nominal, selective and dissociative amnesia.

\section{Therapeutic groups}

The main objective of the Therapeutic Groups is to lead the participants to reflect on work-related issues to help them and equip them to deal with the day-to-day work and the difficulties of professional inclusion. Performed by a Psychologist and with the support of the Social Worker, it is sought in 12 sessions of approximately 1 hour and 30 minutes each, to address topics previously identified by the group as greater need or interest. Among the topics that are always treated in the form of group dynamics are: diversity in companies, motivation, relationship and interpersonal communication, leadership styles, teamwork, coping strategies. The Therapeutic Group is applied in groups of 8 to 12 people, participants of the Insert Program and enables the formation of links, identifications and differentiations, work with the subjectivity and with the singularity of each one of its members. In this way, it enhances dialogical exchanges, the sharing of experiences and the improvement in the adaptation to the individual and collective way of life.

By working to improve social relationships, the levels of knowledge about issues discussed in the group, the ability to deal with situations inherent to the disorder, hope for a more productive and quality life, confidence and emotional relief, are aware of their potentialities, recognize their limitations, understand their own subjectivity, and build a better relationship with themselves and therefore with society.

During these two years between 2016 and 2018, 2 therapeutic groups were performed, and Group 1 had 8 participants, and 4 of them were already starting their labour activities in the formal labour 
market and the other 4 were still unemployed. During Group 1, only 1 participant was removed due to worsening of the symptoms and at the end of the 12 meetings only 1 had not been included in the formal job market. One of the contributions of the Therapeutic Group was to promote the maintenance of the participants in their jobs since the discontinuity in the work activity is common in this population which makes us think about the importance of psychosocial support, listening spaces and dialogical exchanges. After 1 year of the end of the Group 1 meetings, only one participant was unable to remain employed due to worsening of the depressive symptoms and was referred to individual psychotherapy.

A strong point identified in the groups is the extent to which this type of approach improves their socialization and linkage with the Program and strengthens their empowerment in the search for professional inclusion and overcoming the barriers faced since mental illness. Nevertheless, we have identified in participants in the Therapeutic Groups the best response to treatment in PROESQ. They are more motivated to follow the team's recommendations about treatment and committed to the established agreements.

\section{Conclusion}

Schizophrenia has impacts on the functionality of individuals mainly related to global psychosocial mental functions. The elaboration of a work plan for professional inclusion as one of the possible mental health rehabilitation strategies should consider the need to support the overcoming of the deficits and limitations caused by the disorder.

\section{Acknowledgements}

None.

\section{Conflict of interest}

The author declares no conflict of interest.

\section{References}

1. United Nations Convention on the Rights of Persons with Disabilities. USA; 2008:1-28.

2. International Classification of Functions, Disability, and Health (ICF). USA, ASHA, 2004.

3. Presidência da República Casa Civil Subchefia para Assuntos Jurídicos. Brazilian Inclusion Law (13.146/15); 2015. 